\title{
Attomolar SERS detection of organophosphorous pesticides using silver mirror like micro-pyramids as active substrate
}

\author{
Marta Lafuente ${ }^{1,2}$, Erwin J. W. Berenschot ${ }^{3}$, Roald M. Tiggelaar ${ }^{4}$, Sergio G. \\ Rodrigo $^{2,5}$, Reyes Mallada ${ }^{1,2}$, Niels R. Tas ${ }^{3}$, María P. Pina ${ }^{1,2}$
}

${ }^{1}$ Instituto de Nanociencia de Aragón (INA), Departamento de Ingeniería Química y

Tecnologías del Medio Ambiente. Universidad de Zaragoza, 50018, Zaragoza, Spain.

${ }^{2}$ Instituto de Ciencia de los Materiales de Aragón (ICMA), CSIC-Universidad de Zaragoza, 50009, Zaragoza, Spain.

${ }^{3}$ Mesoscale Chemical Systems, MESA+ Institute for Nanotechnology, University of Twente, P.O. Box 217, 7500 AE Enschede, The Netherlands.

${ }^{4}$ MESA+ NanoLab cleanroom, MESA+ Institute for Nanotechnology, University of Twente, P.O. Box 217, 7500 AE Enschede, The Netherlands

${ }^{5}$ Centro Universitario de la Defensa, Carretera Huesca s/n, 50090 Zaragoza, Spain.

María P. Pina, mapina@unizar.es, +34 976751155.

\section{ORCID:}

Marta Lafuente: 0000-0003-2660-3726

Erwin J. W. Berenschot: 0000-0002-4721-2997

Roald M. Tiggelaar: 0000-0002-4021-5036

Sergio G. Rodrigo: 0000-0001-6575-168X

Reyes Mallada: 0000-0002-4758-9380

Niels R. Tas: 0000-0001-7541-4345

María P. Pina: 0000-0001-9897-6527

\begin{abstract}
:
Surface enhanced Raman spectroscopy (SERS) is gaining importance as ultrasensitive analytical tool for routine high throughput analysis of a variety of molecular compounds. One of the main challenges is the development of robust, reproducible and cost-effective SERS substrates. In this work, we study the SERS activity of 3D silver mirror like micro-pyramid structures extended in the z-direction up to $3.7 \mu \mathrm{m}$ (G0 type substrate) or $7.7 \mu \mathrm{m}$ (G1 type substrate), prepared by $\mathrm{Si}$ based microfabrication technologies, for trace detection of
\end{abstract}


organophosphorous pesticides, using paraoxon-methyl as probe molecule. The average relative standard deviation (RSD) for the SERS intensity of the peak displayed at $1338 \mathrm{~cm}^{-1}$ recorded over a centimeter scale area of the substrate is below $13 \%$ for pesticide concentrations in the range $10^{-6}$ to $10^{-15} \mathrm{~mol} \cdot \mathrm{L}^{-1}$. This data underlies the spatial uniformity of the SERS response provided by the microfabrication approach. The minimum detectable concentration of paraoxon-methyl is $10^{-18} \mathrm{~mol} \cdot \mathrm{L}^{-1}$. According to finite-difference time-domain (FDTD) simulations, such remarkable feature is mainly due to the contribution on electromagnetic field enhancement of edge plasmon polaritons (EPPs), propagating along the pyramid edges where the pesticides molecules are preferentially adsorbed.

Keywords: silver-coated micro-pyramids, FDTD simulations, SERS, attomolar detection, pesticides

\section{Introduction}

Pesticide use plays an important role in agricultural production, and in horticulture and forestry, by preventing, destroying, repelling or mitigating any disease and infestation of crops [1]. However, pesticides applied to crops can deteriorate the groundwater and surface ecosystems. Water is the principal access to the food chain and pollution can lead to risk for human and animal health. According to the Agri-environmental indicators for the European Union [2], about of $7 \%$ of ground water drinking water stations and 5\% of river drinking stations reported excessive levels for one or more pesticides in 2013, latest year with available data. The World Health Organization established guidelines for the quality of drinking water regarding the presence of pesticides used in agriculture that, depending on toxicity, could range from 100 to $0.03 \mu \mathrm{g} / \mathrm{L}$ [3]. In addition, the existence of pesticide chemical residues in or on food is becoming a serious concern. Thus, Directive 2009/128/EC aims to achieve a sustainable use of pesticides in the EU. The maximum residue level (MRL) is the highest level of a pesticide residue, normally expressed in ppm or $\mathrm{mg} / \mathrm{kg}$ of food, that is legally tolerated in or on food or feed when pesticides are applied according to good agricultural practice codes. The use of paraoxon-methyl is not allowed by the European commission (Regulation 2003/166/EC, excluding paraoxon from the list in Annex I of Directive 91/414/ECC) due to identified concerns with regard to the safety of operators potentially exposed and the possible impact of the substance on non-target insects, birds and mammals. However, food that is imported from other nations usually has a different violation rate because other countries may allow the use of different pesticides or different amounts of pesticides. 
Accordingly, the development of technologies for detection and identification of trace pesticides is essential for human health [4]. Such technologies have to fulfil well-established characteristics as selectivity, sensibility and reproducibility. Historically, chromatography techniques have been used for pesticides detection, including LC/GC-MS [5], HPLC [6] and TLC [7]. Nowadays, methods for real time and rapid pesticide detection in food are of particular interest for consumers and researchers. Surface-enhanced Raman spectroscopy (SERS) provides not only ultrasensitive and fast detection, but also a rich vibrational spectroscopic information crucial for identification of chemical compounds [8-12].

SERS is based on the enormous enhancement of the Raman signal of molecules adsorbed on suitable metallic nanostructures. Therefore, it could be defined as a combination of two technologies: Raman spectroscopy and nanotechnology [13]. The amplification of the Raman signal of molecules in SERS mainly results from the electromagnetic (EM) enhancement caused by the plasmon resonances generated on a metallic nanostructure. Although SERS has been reported for many metals, the vast majority of studies and applications are developed with gold and silver [13]. In 2D planar metal surfaces or assembled nanoparticles onto flat surfaces, the interaction of the light with the metal substrate produces amplifications of the local EM field due to the metal roughness at the scale of a few nanometers. Molecules confined within these enhanced EM regions suffer a large increase on its cross-section, which produces an increase of its Raman intensity that lowers the limit of detection (LOD) of the molecules. From the point of view of reproducibility, these localized surface plasmons are somehow uncontrolled, due to their random nature. The randomness in SERS detection introduced by 2D mirror-like planar metal surfaces or substrates based on the deposition of metallic nanoparticles, can be avoided with SERS structures supporting extended plasmonic resonances (surface-like), which can be managed by means of a clever design of the enhancing metal surface. Thus, the key point for the widespread use of SERS for analytical applications is to get a reproducible and homogeneous EM effect over large area thanks to the fabrication of uniform, reproducible, affordable and easy to scale-up SERS substrates [14].

The introduction of nanotechnology at the beginning of the $21^{\text {st. }}$ century, with the development of nanofabrication technologies and the possibility to observe and characterize nanostructures with powerful microscopies revolutionized the field of Raman SERS. The preparation of SERS substrates followed either bottom-up or top-down approach. In both cases, there are limitations for the fabrication of homogeneous large areas with precise control of the nanostructures. The development of reliable methodologies for the preparation of homogeneous, robust and cost-effective metallic nanostructure-based SERS substrates remains a field of major interest $[15,16]$. In particular, the degree of control and reproducibility of SERS substrates in terms of performance, process standardization and sample-to-sample reproducibility need to be 
improved [17]. Recently, we have developed SERS active platforms based on 3D-fractal microstructures for the detection of dimethyl methylphosphonate (DMMP), a common surrogate of G-nerve agents [18]. This SERS substrate, containing 3D periodic Ag-mirror like pyramidal micro-structures extended in z-direction up to $12 \mu \mathrm{m}$ height that are coated with a homogeneous monolayer of Au@citrate NPs, displays novel attractive properties in terms of robustness and reproducibility as well as sensitivity.

The aim of this work is trace-level detection of organophosphorous pesticides using noncoated 3D periodic Ag-mirror like pyramidal micro-structures extended in z-direction, up to 7.7 $\mu \mathrm{m}$ as SERS substrates. Such periodic 3D microstructures were fabricated by means of corner lithography and anisotropic wet etching of silicon followed by anodic bonding to glass and dissolvation of the silicon. Subsequently, metallization was performed by electron beam evaporation. 3D-simulations based on the finite-difference time-domain (FDTD) method were performed to gain insight in the excited plasmonic modes and validate the experimentally obtained SERS enhancement factors. Paraoxon-methyl was chosen as target molecule to characterize the SERS detection performance due to its toxicity as pesticide for crop protection.

\section{Experimental}

\section{Materials}

4-nitrobenzenethiol (4-NBT, 80\%) and paraoxon-methyl (analytical standard) were purchased from Sigma-Aldrich. Silver pellets (99.99\%) from Kurt J. Lesker Company, Jefferson Hills, PA, USA were used as metal source for e-beam evaporation.

\section{Fabrication of $\mu$-pyramids}

Two types of 3D pyramidal microstructures, G0 and G1 (see Figure 1), were fabricated by means of corner lithography, anisotropic wet etching of silicon, anodic bonding to glass and silicon removal following a previously described method $[18,19]$. In short, a silicon ( $\mathrm{Si}$ ) wafer with thermally grown $\mathrm{SiO}_{2}$ was patterned in buffered hydrofluoric acid (BHF) using a resist mask with a regular pattern of circular openings ( $5 \mu \mathrm{m}$ diameter and $25 \mu \mathrm{m}$ periodicity). The un-masked silicon was anisotropically etched in potassium hydroxide $(\mathrm{KOH})$ in order to create inverted pyramidal-shaped pits. Afterwards, the remaining oxide mask was stripped. Next, the wafer was uniformly coated low-stress silicon-rich silicon nitride (SiRN; 160nm). At this point, the inverted pits for making active substrates type G0 were finished (Figure 1a). To create inverted microstructures required for obtaining active substrates type G1, the processing continued with immersion in hot phosphoric acid $\left(\mathrm{H}_{3} \mathrm{PO}_{4}\right)$ to isotropically etch the SiRN (Figure 1b) until only a dot in the corner of the inverted pyramid was remaining (Figure 1c). This step is called corner lithography. The following step was local oxidation of silicon 
(LOCOS process), during which exposed silicon was locally oxidized using the SiRN dots as mask. The silicon nitride in the corner of each inverted micropyramids was stripped with $\mathrm{H}_{3} \mathrm{PO}_{4}$. Next, the un-masked silicon in the apex was etched anisotropically using tetramethyl ammonium hydroxide (TMAH), which formed an octahedral shaped feature at the tip of each inverted pyramid (Figure 1d). After stripping of $\mathrm{SiO}_{2}$ and deposition of $160 \mathrm{~nm} \mathrm{SiRN}$ the inverted microstructures for realizing substrates type G1 were finished. This fabrication technique allows the fabrication of SERS substrate in large scale (wafer-scale), reducing the cost of the process. In order to acquire substrate types G0 and G1 -i.e. surfaces textured with periodic, hollow microstructures composed of SiRN anodic bonding of the processed silicon wafer to a Mempax glass wafer ( $500 \mu \mathrm{m}$ thick) was performed, followed by dissolvation of the silicon. Thus, both G0 and G1 type substrates contain hollow microstructures upright positioned on a glass plate. The height of the microstructures in case of a G0 type substrates is $3.7 \mu \mathrm{m}$, whereas in case of a G1 type substrate the height of the microstructures is $7.7 \mu \mathrm{m}$.

Following the fabrication, G0 and G1type $\mu$-pyramids were metallized with 40nm of silver via electron beam evaporation (Edwards auto-500). The conditions during the evaporation were $3 \times 10^{-7} \mathrm{mbar}, 32 \mathrm{~mA}$ and $5.3 \mathrm{kV}$.

\section{Materials characterization}

Scanning electron microscopy (SEM) images were recorded using a FEI INSPECT 50 ( $20 \mathrm{kV}$ and spot size 3.5). The Image $\mathrm{J}$ analysis software was used to evaluate the characteristic features of the G0 and G1 pyramids, and the silver thickness on the substrate from various TEM images of lamella G0 and G1 silver-coated microstructures prepared using focus-ion beam (FIB). G0 and G1 specimen were cut in transversal direction. TEM images were recorded using a FEI Tecnai T20 and the FIB equipment was a Dual Beam Helios Nanolab. The roughness of the Ag layer was measured by Atomic force microscopy (AFM; Multimode 8 from VeecoBruker; tip, OMCL-AC240TN-W2 from OLYMPUS). Measurements were conducted in tapping mode in air. Roughness was estimated by analysis of topography images with Gwyddion 2.45.

\section{Raman and SERS measurements}

The Raman spectra were acquired with a confocal Alpha300 Raman spectrophotometer from WITec with spectral resolution of $2 \mathrm{~cm}^{-1}$. All measurements were made in backscattering geometry with an excitation wavelength of $785 \mathrm{~nm}$, focusing the sample with a $20 \mathrm{x}$ microscope objective and applying a power of $5 \mathrm{~mW}$ for $1 \mathrm{~s}$. Five different maps of $200 \mu \mathrm{m} \times 150 \mu \mathrm{m}$ containing $10 \times 10$ spots were measured on the 2D planar and 3D G0 and G1 type substrates, 
respectively. The planar substrates consist in a flat glass surface (without the pyramids) coated with $40 \mathrm{~nm}$ of silver. Raman spectrum of liquid solution $\left(9 \times 10^{-3} \mathrm{M}\right)$ of 4-nitrobenzenethiol was measured using 5mW and 50s. In all SERS and Raman spectra automatic baseline (background) has been subtracted by WITec Software 2.10. The analytical enhancement factor has been calculated according to methods established in the literature [20]. Details about the calculation are presented in the Supporting Information S1.

\section{Detection of organophosphorous pesticides}

In this work, the stock solution of paraoxon-methyl $\left(1 \times 10^{-3} \mathrm{M}\right)$ was prepared in ethanol. Then, paraoxon solutions with concentrations of $10^{-6} \mathrm{M}, 10^{-9} \mathrm{M}, 10^{-12} \mathrm{M}, 10^{-15} \mathrm{M}$ and $10^{-18} \mathrm{M}$ were prepared by step-by-step dilution with the same solvent.

For SERS measurements, the substrates $\left(0.2 \mathrm{~cm}^{2}\right)$ were incubated in $25 \mathrm{~mL}$ of the adequate concentration of paraoxon in vertical position for 40 minutes. After that, the SERS substrates were rinsed in ethanol and air-dried. The average of five SERS mappings (200 $\mu \mathrm{m} \times 150 \mu \mathrm{m}$ containing $10 \times 10$ spots) acquired over a centimeter scale area is presented. The signals of paraoxon-methyl at $746 \mathrm{~cm}^{-1}$ and $1338 \mathrm{~cm}^{-1}$, corresponding to $\mathrm{NO}_{2}$ scissor and symmetric stretching of $\mathrm{NO}_{2}$, respectively.

\section{Finite-difference time-domain (FDTD) simulations}

A homemade code of the finite-difference time-domain (FDTD) method [21] was used to compute EM fields in the fabricated G0 and G1 type substrates. For all simulations the $\mu$ pyramids were considered as isolated: the distance between adjacent $\mu$-pyramids in the fabricated samples prevents direct or indirect re-illumination. Within this approximation, the computational domain was terminated with absorbing boundary conditions in order to avoid spurious back-reflections. The dielectric constant of silver was taken from tabulated data and incorporated to FDTD through a Drude-Lorentz model [22]. A linearly polarized Gaussian beam illuminates the structures at normal incidence. The beam waist at the structures is 2 microns, similar to the laser spot size at the experimental sample. A mesh size small enough to represent faithfully the EM fields inside the metal was used (see [21], for further details).

Average SERS gain for a given structure $S, \alpha_{S}$, and for a flat metal surface $M, \alpha_{M}$, is defined as:

$$
\begin{aligned}
& \alpha_{S}=\left\langle\left|\frac{\vec{E}_{S}(\vec{r}, \lambda)}{\vec{E}_{0}(\vec{r}, \lambda)}\right|^{4}\right\rangle \\
& \alpha_{M}=\left\langle\left|\frac{\vec{E}_{M}(\vec{r}, \lambda)}{\vec{E}_{0}(\vec{r}, \lambda)}\right|^{4}\right\rangle
\end{aligned}
$$


where the brackets indicate average integration over the SERS generating volume [21]. In the numerical implementation of Equation 1, the electric field intensity at the metal surface (the source of SERS) is approximated as the averaged $\overline{\boldsymbol{E}}_{S}$ and $\overline{\boldsymbol{E}}_{\boldsymbol{M}}$ fields within each single mesh cell in contact with the metal. The incident field $\overline{\boldsymbol{E}}_{\boldsymbol{0}}$ is also obtained in vacuum at the same position $\overrightarrow{\boldsymbol{r}}$ and wavelength $\lambda$, to finally reproduce the local SERS intensity.

\section{Results and discussion}

\section{Morphological characterization of silver micro-pyramids}

Figure 1 shows SEM images of the top view and cross-section of the obtained substrates, which evidences the reproducibility of the fabrication process for the pyramidal type microstructures. The thickness of the continuous silver layer was determined by means of a TEM lamella (see Figure S2 in SI) and was found to be $40 \pm 7 \mathrm{~nm}$ for G0 pyramids and $45 \pm 6 \mathrm{~nm}$ for G1 microstructures, respectively. Figure $1 \mathrm{~g}$ and $\mathbf{j}$ show schematics of the silver layer evaporation. The average roughness was measured by AFM and found to be in $2.6 \pm 0.2$ $\mathrm{nm}[18]$.

(a)

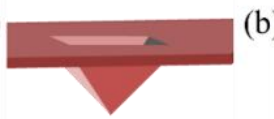

G0 pyramid (c)

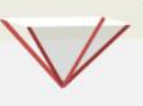

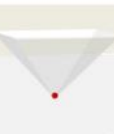

(d)

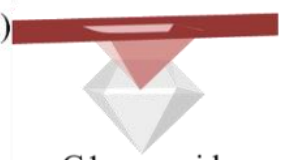

G1 pyramid

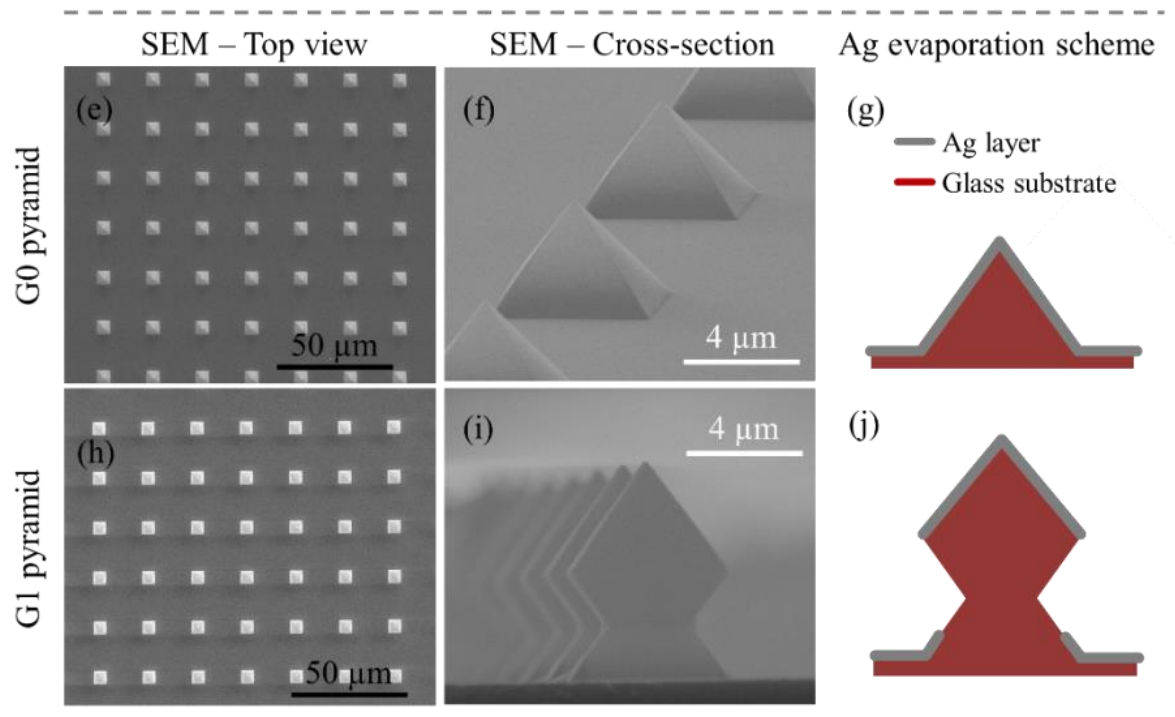

Figure 1 Scheme of G0 and G1 type substrate fabrication based on inverted pyramidal and octahedral microstructures: the evolution from G0 (a) to G1 (d). Top view (e,h) and cross-section (f,i) SEM images of silver-coated G0 and G1 type substrates. (g,j) schemes of G0 and G1 type micro-pyramids after silver evaporation.

\section{SERS activity of silver-coated micro-structures}


The SERS signal of the 4-nitrobenzenethiol (4-NBT) was studied in order to evaluate the SERS activity of the substrate. 4-NBT is a well-known SERS target analyte used for the quantification and inter-comparison of enhancement factors. We first investigated the capability of the plasmonic SERS substrates to increase the Raman signal. Optical microscope images of the SERS mapping area, are presented together with SERS mapping intensities of the $\mathrm{NO}_{2}$ stretching peak at $1338 \mathrm{~cm}^{-1}$ of 4-NBT, in Figure 2a and b, respectively. The SERS mapping clearly allows distinguishing rows with micro-structures (bright area) from parts without structures, i.e. flat surfaces (dark area), which perfectly matches with the optical microscope images of the same area (Figure 2). The uniformity of the pyramid pattern in the large area scanned (area $200 \mu \mathrm{m} \times 150 \mu \mathrm{m} ; 10 \times 10$ points), together with hot spots located in the pyramids allows us to average the signal in the whole area, resulting in an average spectra that it is similar in the 5 different mappings randomly selected over the centimeter scale surface area of the substrate (see Figure S1). Averaging the peak at $1338 \mathrm{~cm}^{-1}$, in the 5 areas resulted in a value of $1389 \pm 185 \mathrm{cts}$ corresponding to a $13 \%$ RSD. This methodology results in a reproducible signal with low standard deviation which is one of the challenges in SERS for detection and quantification. Figure 2c shows these average spectra for 3D G0 and G1 type substrates as well as the spectrum obtained on a 2D planar substrate covered with silver and the reference spectrum of 4-NBT in solid phase. The characteristic peaks of 4-NBT at $747 \mathrm{~cm}^{-1}(\mathrm{C}-$ $\mathrm{S}$ stretching), $1338 \mathrm{~cm}^{-1}\left(\mathrm{NO}_{2}\right.$ stretching) and $1532 \mathrm{~cm}^{-1}$ (C-C stretching) are clearly observed on G0 and G1 type substrates, in contrast to the flat substrate. The intensity of the signal is clearly higher in the case of G1. From the SERS mappings, the analytical enhancement factor $(\mathrm{AEF})$ is calculated using Equation S2. The AEF was $1.3 \times 10^{5}$ and $6.4 \times 10^{5}$ for G0 and G1 type substrates, respectively. Besides, the ratio of $\mathrm{AEF}_{\mathrm{G} 1} / \mathrm{AEF}_{\mathrm{G} 0}$ accounts circa $\sim 5$. It is important to note that this AEF corresponds to the averaged signal in the whole area. If we consider only the enhancing surface according to FDTD simulations as explained in Figure 6, the AEF would increase one order of magnitude.
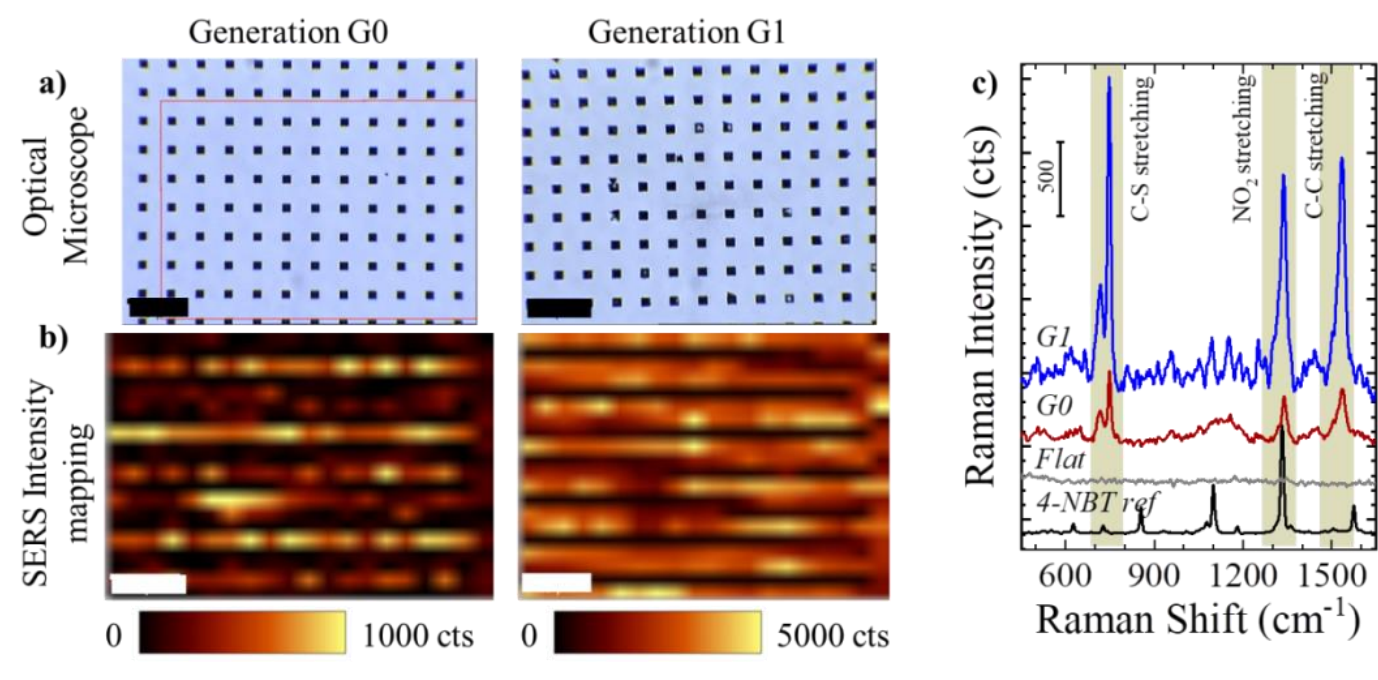
Figure 2 (a) Optical microscope images of SERS substrates with G0 and G1 silver pyramids. Scale bar $40 \mu \mathrm{m}$; (b) SERS mapping obtained at $1338 \mathrm{~cm}^{-1}$ for SERS substrates with G0 and G1 silver pyramids. Scale bar $40 \mu \mathrm{m}$; (c) The average SERS spectrum of 4-NBT $10^{-6} \mathrm{M}$ obtained in each mapping of G0 (red) and G1 (blue) type substrates. The homologous spectrum acquired on a flat substrate with 40 $\mathrm{nm}$ of silver is also included as control. The normal Raman was acquired on 4-NBT in solid phase.

Yellow/grey-marked zones indicate the main vibrational modes of 4-NBT.

\section{Application of Ag micro-pyramids as SERS substrates to paraoxon detection}

Following the same methodology as in the case of 4-NBT, the SERS activity of the G1 type substrate was tested on pesticide detection using paraoxon as target molecule (Figure 3a). The averaged SERS spectra for the different concentrations of paraoxon are shown in Figure 3b. For the complete concentration range $\left(10^{-6}-10^{-18} \mathrm{M}\right)$, the characteristic fingerprint of the paraoxon is clearly distinguished, i.e. a total of ten vibrational modes, labelled $a-j$ in Figure 3. The complete assignation of bands is given in Table 1. Figure 3 also shows that the relative intensity of the peaks related to the $\mathrm{NO}_{2}$ group differ from the acquired at normal Raman conditions. The peaks involved in this change of intensities are $634 \mathrm{~cm}^{-1}$ (band $\mathrm{b}-\mathrm{NO}_{2}$ scissor), $746 \mathrm{~cm}^{-1}$ (band $\mathrm{c}-\mathrm{NO}_{2}$ scissor), $1145 \mathrm{~cm}^{-1}$ (band $\mathrm{e}-\mathrm{NO}_{2}$ asymmetric stretching), $1338 \mathrm{~cm}^{-1}$ (band $\mathrm{g}-\mathrm{NO}_{2}$ symmetric stretching) and $1532 \mathrm{~cm}^{-1}$ (band $\mathrm{i}-\mathrm{NO}_{2}$ unsymmetric stretching). A possible explanation relies on the preferential interaction of paraoxon molecules with metallic silver mediated by the partially positive charge on the nitro functional groups.

a)
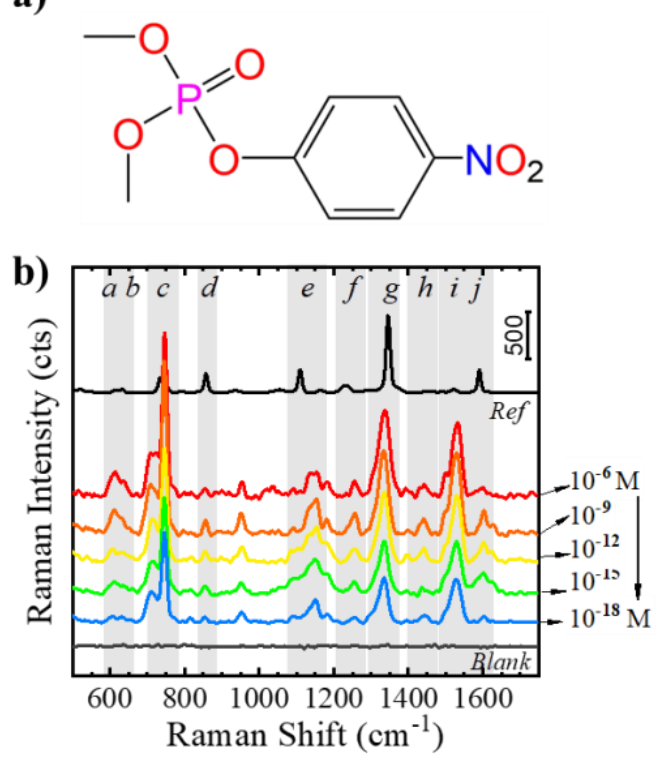

Figure 3 (a) Paraoxon-methyl molecule. (b) SERS spectra of different concentrations $\left(10^{-6} \mathrm{M}\right.$ to $10^{-21}$ M) of paraoxon collected on G1 type substrate, together with the Raman spectrum of neat paraoxon in solid phase. Grey-marked zones indicate the main vibrational modes. See Table 1 for more details.

A detailed analysis of the SERS response as a function of the paraoxon-methyl concentration is presented in Figure 4a-b showing the SERS intensity mappings of two different peaks of paraoxon methyl, i.e. $\mathrm{NO}_{2}$ scissor (band $\mathrm{c}-746 \mathrm{~cm}^{-1}$ ) and $\mathrm{NO}_{2}$ symmetric stretching (band $\mathrm{g}$ - 
$1338 \mathrm{~cm}^{-1}$ ), as a function of concentration. As it can be observed, for both peaks the SERS intensity decreases when the concentration of pesticide is reduced from $10^{-6} \mathrm{M}$ to $10^{-18} \mathrm{M}$. These mappings also reveal a very good spatial resolution matching with the spatial distribution of the micropyramids on the 3D G1 type substrate. As expected, the density of SERS signal lowered for diluted concentrations of paraoxon due to a smaller number of molecules adsorbed on the substrate upon immersion in lower concentrations. In the case of $10^{-18} \mathrm{M}$, approximately $15 \times 10^{3}$ molecules are present in the solution during incubation. Assuming that all the molecules are homogeneously distributed on the surface of the SERS substrate, an average of 23 molecules per scanned area can be expected (see calculations in the supporting information S3). This is consistent with the few spots observed with the confocal Raman at $10^{-18} \mathrm{M}$ (see Figure 4a and Figure $4 \mathrm{~b}$ ). However, as the concentration increases a full monolayer of paraoxon molecules is expected, up to covering the whole lateral surface area of the pyramids at a concentration around $10^{-12} \mathrm{M}$. A further increase in the pesticide concentration leads to an almost invariant SERS intensity because such additional molecules are located on the colder 2D planar metal surface.

Furthermore, the reproducibility of the signal obtained on G1 type substrates was investigated. The average SERS intensity of paraoxon peaks displayed at $746 \mathrm{~cm}^{-1}$ and $1338 \mathrm{~cm}^{-}$ ${ }^{1}$ recorded at five different areas for two different concentrations is shown in Figure $4 \mathbf{c}\left(10^{-15}\right.$ M) and in Figure 4d $\left(10^{-18} \mathrm{M}\right)$. As can be observed, the SERS signal was higher at $746 \mathrm{~cm}^{-1}$ (band $\mathrm{c}$ of Figure 3). The relative standard deviation (RSD) of the five measurements for paraoxon $10^{-18} \mathrm{M}$ at $746 \mathrm{~cm}^{-1}$ and $1338 \mathrm{~cm}^{-1}$ was $26 \%$ and $24 \%$, respectively (Figure 4c).

Table 1 Tentative assignment of the Raman bands for paraoxon on G1type substrates. Band identification corresponds with those highlighted in grey on Figure 3.

\begin{tabular}{|c|c|c|}
\hline $\begin{array}{l}\text { Band } \\
\text { ID. }\end{array}$ & $\begin{array}{c}\text { Raman } \\
\text { Shift } \\
\left(\mathrm{cm}^{-1}\right)\end{array}$ & Vibrational mode [23] \\
\hline $\mathrm{a}$ & 612 & Out of plane ring movement \\
\hline $\mathrm{b}$ & 634 & $\mathrm{NO}_{2}$ scissor, $\mathrm{CC}$ bending \\
\hline $\mathrm{c}$ & 746 & $\mathrm{NO}_{2}$ scissor, $\mathrm{CC}$ bending \\
\hline d & 856 & $\mathrm{NO}_{2}$ scissor $\left(\mathrm{Ar}-\mathrm{NO}_{2}\right)$ \\
\hline $\mathrm{e}$ & 1145 & $\begin{array}{l}\mathrm{CH} \text { bend (in plane) / } \mathrm{NO}_{2} \\
\text { asymmetric stretch }\end{array}$ \\
\hline $\mathrm{f}$ & 1256 & $\mathrm{P}=\mathrm{O}$ stretch \\
\hline g & 1338 & Symmetric stretching $\mathrm{NO}_{2}$ \\
\hline $\mathrm{h}$ & 1443 & Ring deformation \\
\hline $\mathrm{i}$ & 1532 & Unsymmetric stretching $\mathrm{NO}_{2}$ \\
\hline $\mathrm{j}$ & 1590 & Phenyl ring vibration \\
\hline
\end{tabular}




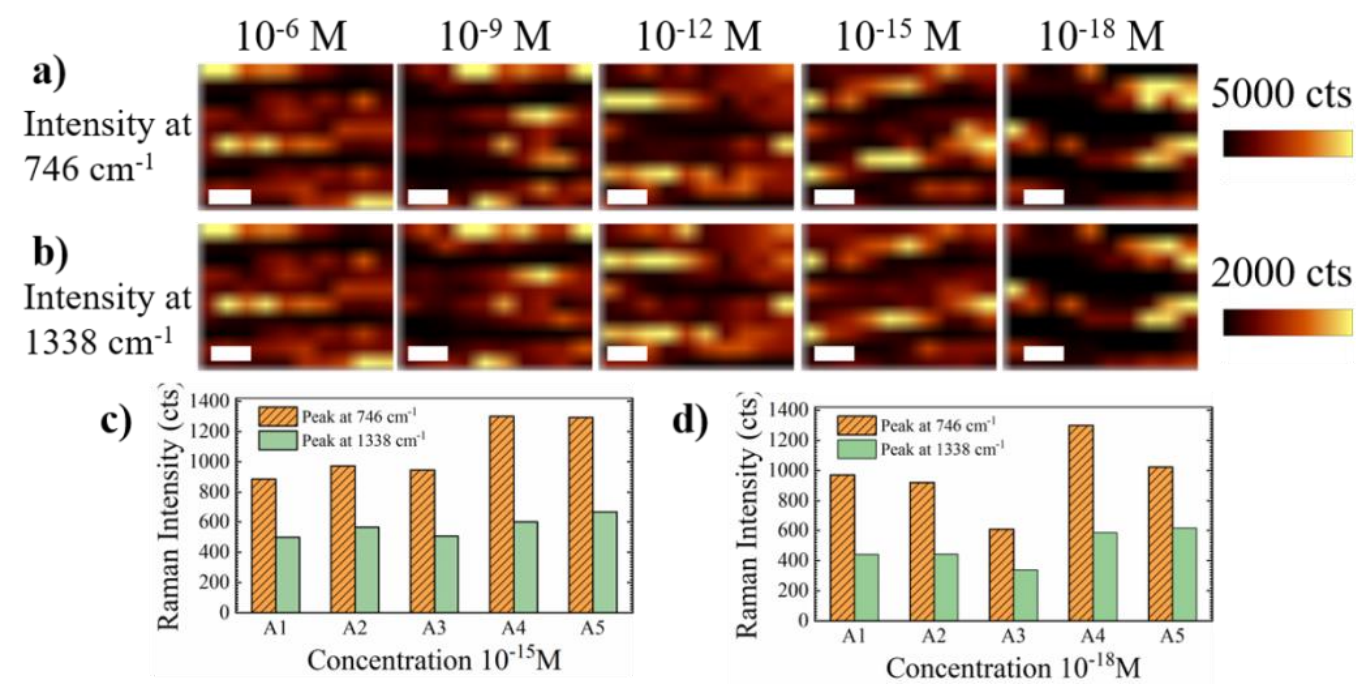

Figure 4 SERS mappings recorded for different concentrations of paraoxon on G1 type substrate, monitoring peak at (a) $746 \mathrm{~cm}^{-1}$ and (b) $1338 \mathrm{~cm}^{-1}$. The average SERS intensity of each paraoxon peak obtained at concentrations of (c) $10^{-15} \mathrm{M}$ and (d) $10^{-18} \mathrm{M}$ in the five different recorded areas.

The relevance of these results is revealed in Table 2, where we compared the obtained experimental LOD with the available data in literature for paraoxon pesticide with SERS active substrates. The experimental LOD, $10^{-18} \mathrm{M}$, is the lowest reported, being three orders of magnitude lower than the best reported value $\left(4 \times 10^{-15} \mathrm{M}\right)$. These results confirmed the exceptional performance of the 3D G1 type SERS substrates for SERS detection developed in this work.

Table 2 Comparison of detection of paraoxon with different SERS substrates.

\begin{tabular}{|c|c|c|c|c|c|}
\hline Material & Detection / Methodology & $\begin{array}{l}\text { Raman } \\
\text { Methodology }\end{array}$ & $\begin{array}{l}\text { Minimum } \\
\text { concentration } \\
\text { experimentally } \\
\text { measured } \\
\end{array}$ & RSD & Reference \\
\hline G1 type substrate & $\begin{array}{l}\text { Direct / Incubation the } \\
\text { SERS substrate with the } \\
\text { analyte solution }\end{array}$ & $\begin{array}{l}\text { Mapping } \\
\text { mode } \\
\text { averaging } \\
300 \text { spectra } \\
\text { over cm } \\
\text { scale area }\end{array}$ & $10^{-18} \mathrm{M}$ & $\begin{array}{l}< \\
26 \%\end{array}$ & This work \\
\hline AuNPs & $\begin{array}{l}\text { Indirect via } \\
\text { acetylcholinesterase / } \\
\text { Mixing the analyte } \\
\text { solution with the AuNP } \\
\text { solution. }\end{array}$ & 5 spectra & $4 \times 10^{-15} \mathrm{M}$ & $5 \%$ & [24] \\
\hline Au@AgNPs & $\begin{array}{l}\text { Direct / Mixing the } \\
\text { analyte solution with the } \\
\text { AuNP solution. }\end{array}$ & 3 spectra & $10^{-9} \mathrm{M}$ & $10 \%$ & {$[25]$} \\
\hline
\end{tabular}




\begin{tabular}{llllll}
\hline Polystyrene/AgNPs & $\begin{array}{l}\text { Direct / Adding a drop of } \\
\text { the analyte solution onto } \\
\text { the SERS substrate. }\end{array}$ & 3 spectra & $2 \times 10^{-7} \mathrm{M}$ & N.A. & [26] \\
\hline Ag nanocubes & $\begin{array}{l}\text { Direct / Mixing the } \\
\text { analyte solution with the } \\
\text { AuNP solution. }\end{array}$ & Not available & $10^{-8} \mathrm{M}$ & N.A. & [27] \\
\hline $\begin{array}{l}\text { Graphene oxide - } \\
\text { AgNPs }\end{array}$ & $\begin{array}{l}\text { Direct / Adding a drop of } \\
\text { the analyte solution onto } \\
\text { the SERS substrate. }\end{array}$ & Not available & $10^{-7} \mathrm{M}$ & N.A. & [28] \\
\hline
\end{tabular}

\section{Origin of attomolar pesticide detection with G1 type SERS substrates}

One intriguing question is the smooth evolution of SERS intensity with concentration observed in Figure 3 and Figure 4. This is clearly seen in Figure 5Error! Reference source not found. that shows the average SERS intensity of peaks at $746 \mathrm{~cm}^{-1}$ and $1338 \mathrm{~cm}^{-1}$ at different concentrations of paraoxon. We attributed the two distinct SERS trends observed in that figure to the spatial distribution of target molecules and the electromagnetic field density distribution on G1 type substrates, as explained next.

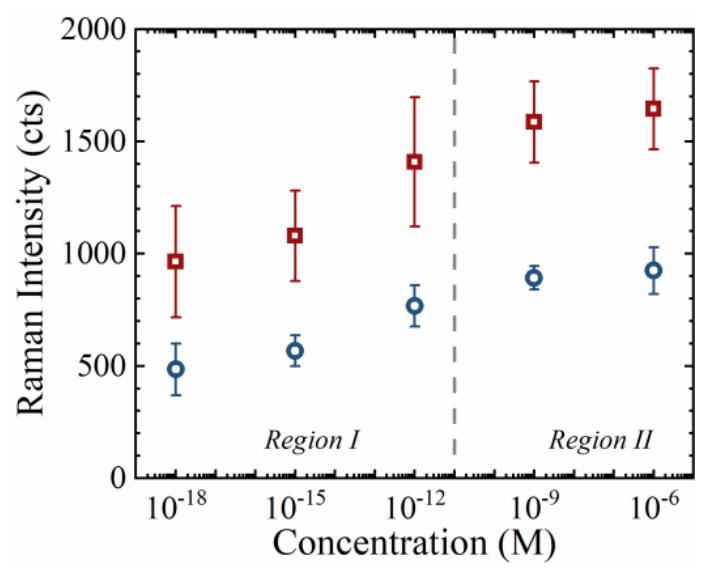

Figure 5 SERS intensity at $746 \mathrm{~cm}^{-1}$ (red square) and $1338 \mathrm{~cm}^{-1}$ (blue circle) as a function of pesticide concentration.

The outperforming of pyramidal type substrates results from the excitation of subwavelength confined electromagnetic (EM) resonances, not present in 2D planar metal substrates. To elucidate the nature of such EM modes, FDTD simulations were carried out. Figure 6a is an example of such calculations, where the intensity of the electric field at $785 \mathrm{~nm}$ wavelength is shown, for two different cross-cuts of the G1 type pyramid. From numerical simulations like that, the average of SERS gain for 3D substrates types G0 and G1 can be calculated with Equation 1. The results for visible and near infrared wavelengths are shown in Figure 6b (bottom). To compare the quality of the different structures as SERS substrates, integration was done only over the metallic pyramid (Figure 6a, grey surface), neglecting so the contribution from the flat metal surface surrounding the microstructures. 
The spectral response of both pyramidal microstructures consists in a sequence of peaks over a background, very similar for both pyramids. On average, SERS gain is slightly higher for G1 than for G0 and the peaks better defined in the first case, within the spectral window shown. The ratio between these values is 1.5 at most, which confirms the experimental results with 4NBT molecules, that G1 based SERS substrates performs alike the G0 ones in the spectral range investigated here. However, calculations at the infrared (not shown) predicts that the G1 pyramid would take over G0 in terms of SERS gain, once the wavelength of incident light is comparable to the pyramid size. For comparison, the corresponding value for a flat metal surface is also shown in Figure 6b (top) with a green line (the area of integration for the flat surface corresponds with the area that the pyramids projects onto the metal surface). Like in the experimental results, this value is much lower, up to two orders of magnitude, than the one found for micro-pyramid substrates.

The agreement between theory and experiments is quite good, attending the typical difficulties that these studies typically face [29]. Interestingly, SERS is low in both AEF experiments and SERS gain calculations. However, why modest SERS values can produce: i) clear differences in SERS between pyramids and flat surface, and ii), detection at ultra-low concentrations.

We elucidate that both results are due to the excitation of Edge Plasmon Polaritons (EPP), which provide the necessary EM field enhancement, creating controllable "hot-regions" at the pyramid edges, by proper design. Figure 6c shows the SERS gain distribution on the G1 pyramid surface, for three different wavelengths. The highest values of SERS gain occur along the edges, as a response to the excitation of standing EM waves of plasmonic origin. The whole pyramid, and in particular the edges, act as an optical cavity. As expected, the index mode of the cavity (which can be labelled by the number of nodes of the standing wave) decreases as the wavelength increases [30]. The standing wave nature of EPP modes is illustrated above the panels. The lines represent the EM in a single edge of the micro-pyramid. There is not only EPP modes excited, the so-called breathing modes are also seen in Figure 6c, displaying complex near-field patterns on the surface. Breathing modes contribute less than EPPs to SERS. The small squares in panel c) correspond to averaged SERS gain values on G1 (top) and flat silver (bottom), taken from panel (b), and represented as top-views, with same scale that the main plots. Clearly, the average SERS gain in the G1 pyramid does not faithfully represent the distribution on the surface of the EM field. 

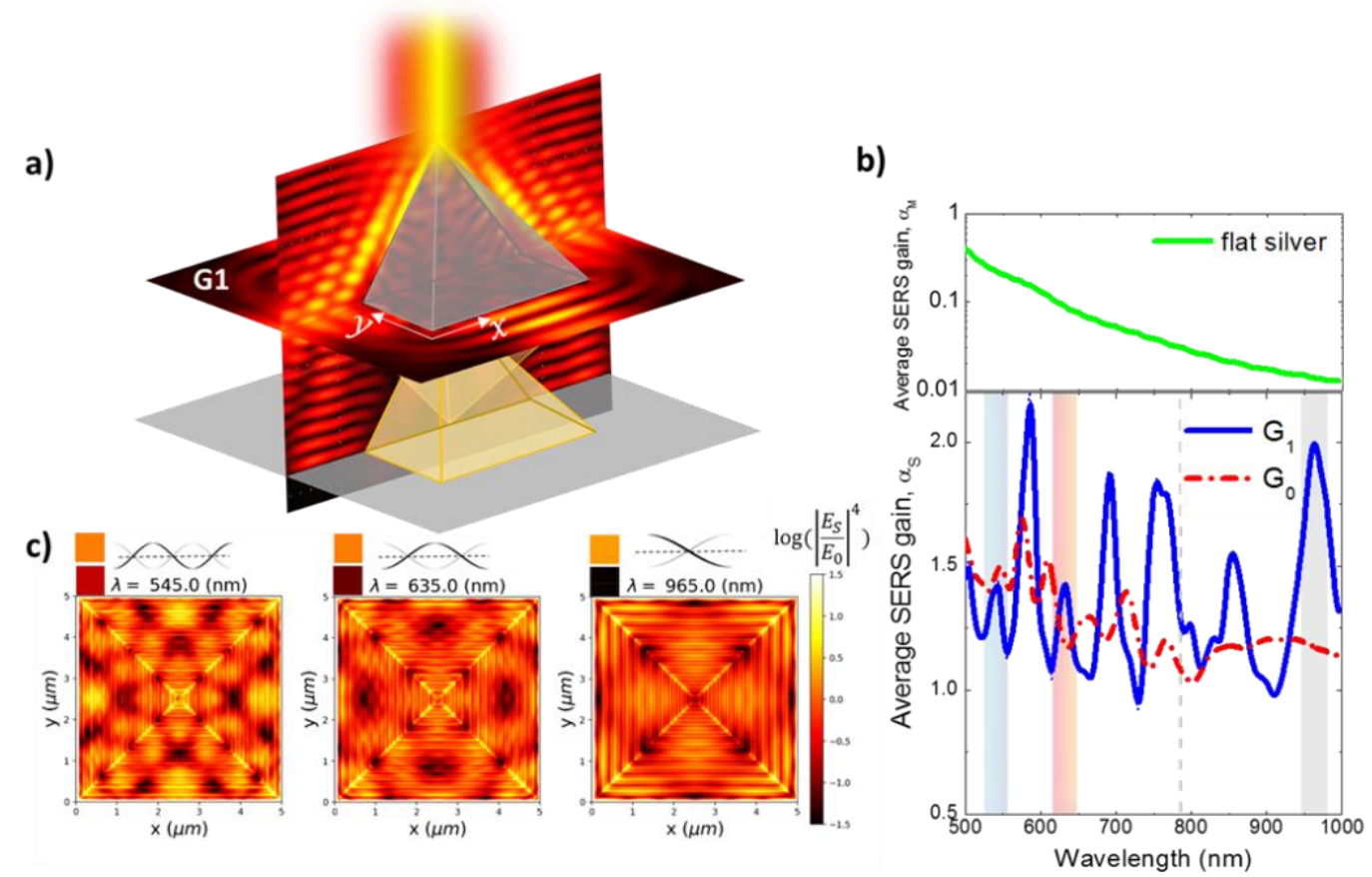

Figure 6 (a) Intensity of the electric field at $785 \mathrm{~nm}$ wavelength for the G1 type pyramid and two different cross-cuts. (b) Bottom: average SERS gain as calculated with Equation 1 for both Ag-coated microstructures: G0 (red colour) and G1 (blue colour). For comparison, the corresponding value for a flat metal surface is shown, with a green line (top). The area of integration for the flat surface corresponds with the area that the pyramids projects onto the metal surface. (c) The logarithm SERS gain distribution on the surface of the G1 pyramid, for three different plasmonic resonances (indicated by the shaded regions in panel (b)). The standing wave nature of EPP modes is illustrated by the lines shown above the panels. Colour squares corresponds to G1 (top) and flat silver (bottom) averages from panel (b), represented with same scale that the main plots.

To sum up, at low pesticide concentrations, the logarithmic SERS response is characteristic of the paraoxon molecules located on the highly SERS active micro-pyramid edges. The preferential analyte adsorption on the apex and pyramidal edges is thermodynamically driven to minimize the free surface energy imposed by the high number of grain boundary defects. Each added molecule in the concentration range from $10^{-18}$ to $10^{-12} \mathrm{~mol} \cdot \mathrm{L}^{-1}$, denoted as Region $\mathrm{I}$ in Figure 5, is preferentially adsorbed on the SERS active sites located on the pyramidal edges (accounting for less than $0.1 \%$ of the scanned area) where the local EM field density is at maximum, and lateral faces (breathing modes accounting for less than $10 \%$ of the scanned area). Considering $0.2 \mathrm{~nm}^{2}$ as the cross sectional area of paraoxon [31], the complete formation of a monolayer on the lateral surface area of the micropyramids would be attained at $\sim 10^{-11} \mathrm{~mol} \cdot \mathrm{L}^{-1}$ (see supporting information S3 and S4 for more detailed calculations). Thus, when increasing concentration, from $10^{-11}$ to $10^{-6} \mathrm{~mol} \cdot \mathrm{L}^{-1}$, a second logarithmic behaviour is shown. The slope is weaker than in Region I for highly diluted pesticide concentrations. In this Region II, each new added molecule is adsorbed on colder, i.e. less active, SERS sites allocated on the planar 2D 
metal surface (accounting for $90 \%$ of the scanned area), which are contributing in much less extent to the total registered SERS signal.

\section{Conclusions}

A large area SERS substrate comprising silver mirror like micro-pyramid structures has been successfully fabricated, modelled and demonstrated for pesticide detection in synthetic samples. The periodic silver-coated (40 nm layer thickness) microstructures fabricated by corner lithography followed by silver evaporation, constitute a robust and reproducible alternative for the development of cost-effective ultrasensitive SERS substrates. The complete Raman fingerprint of paraoxon-methyl is recorded for all studied concentrations in the range $10^{-6}$ to $10^{-}$ ${ }^{18} \mathrm{~mol} \cdot \mathrm{L}^{-1}$. The methodology employed mapping a large area and averaging the signal in the whole area results in a low RSD value, $<11 \%$ for the $10^{-6}$ to $10^{-9} \mathrm{M}$ concentration range where a full coverage of the 3D G1 type substrate with molecules is achieved. These concentrations are within the range of admissible values for pesticides in drinking water according to the WHO. In the case of lower pesticide concentrations, above $10^{-15} \mathrm{M}$, the RSD remains below $20 \%$.

Acknowledgments Authors are grateful for financial support MICINN (CTQ2013-49068-C2-1R, CTQ2016-79419-R and MAT2017-88358-C3-2-R), Gobierno de Aragón (T57-17R p) and Feder 2014-2020 “Construyendo Europa desde Aragón". The microscopy images have been recorded in the Laboratorio de Microscopias Avanzadas at Instituto de Nanociencia de AragonUniversidad de Zaragoza. Authors acknowledge the LMA-INA for offering access to their instruments and expertise.

Compliance with ethical standards The authors declare that they have no competing interest.

\section{References}

1. European Environment Agency. https://www.eea.europa.eu/airs/2018/environment-andhealth/pesticides-sales. Accessed 20 Sep 2018

2. Agri-environmental indicators. https://ec.europa.eu/eurostat/statisticsexplained/index.php?title=Agri-environmental_indicators. Accessed 20 Sep 2019

3. World Health Organization (2017) Guidelines for Drinking-water Quality: fourth edition incorporating the first addendum. Geneva

4. Carvalho FP (2006) Agriculture, pesticides, food security and food safety. Environ Sci Policy 9:685-692. https://doi.org/10.1016/j.envsci.2006.08.002 
5. Hernández F, Sancho J V., Pozo OJ (2005) Critical review of the application of liquid chromatography/mass spectrometry to the determination of pesticide residues in biological samples. Anal Bioanal Chem 382:934-946. https://doi.org/10.1007/s00216005-3185-5

6. Aulakh JS, Malik AK, Kaur V, Schmitt-Kopplin P (2005) A review on solid phase micro extraction - High performance liquid chromatography (SPME-HPLC) analysis of pesticides. Crit Rev Anal Chem 35:71-85. https://doi.org/10.1080/10408340590947952

7. Sherma $\mathbf{J}$ (2015) Review of advances in the thin layer chromatography of pesticides: 2012-2014. J Environ Sci Heal - Part B Pestic Food Contam Agric Wastes 50:301-316. https://doi.org/10.1080/03601234.2015.1000163

8. Pang S, Yang T, He L (2016) Review of surface enhanced Raman spectroscopic (SERS) detection of synthetic chemical pesticides. TrAC - Trends Anal Chem 85:73-82. https://doi.org/10.1016/j.trac.2016.06.017

9. Jiang Y, Sun DW, Pu H, Wei Q (2018) Surface enhanced Raman spectroscopy (SERS): A novel reliable technique for rapid detection of common harmful chemical residues. Trends Food Sci Technol 75:10-22. https://doi.org/10.1016/j.tifs.2018.02.020

10. Wang R, Xu Y, Wang R, et al (2017) A microfluidic chip based on an ITO support modified with $\mathrm{Ag}-\mathrm{Au}$ nanocomposites for SERS based determination of melamine. Microchim Acta 184:279-287. https://doi.org/10.1007/s00604-016-1990-5

11. Ren X, Cheshari EC, Qi J, Li X (2018) Silver microspheres coated with a molecularly imprinted polymer as a SERS substrate for sensitive detection of bisphenol A. Microchim Acta 185:. https://doi.org/10.1007/s00604-018-2772-z

12. Jiang J, Ma L, Chen J, et al (2017) SERS detection and characterization of uranyl ion sorption on silver nanorods wrapped with Al2O3 layers. Microchim Acta 184:27752782. https://doi.org/10.1007/s00604-017-2286-0

13. Stiles PL, Dieringer JA, Shah NC, Van Duyne RP (2008) Surface-enhanced Raman spectroscopy. Annu Rev Anal Chem 1:601-626. https://doi.org/10.1146/annurev.anchem.1.031207.112814

14. Langer J, Jimenez de Aberasturi D, Aizpurua J, et al (2019) Present and Future of Surface-Enhanced Raman Scattering. ACS Nano. https://doi.org/10.1021/acsnano.9b04224

15. Mosier-Boss P (2017) Review of SERS Substrates for Chemical Sensing. Nanomaterials 7:142. https://doi.org/10.3390/nano7060142 
16. Fan M, Andrade GFS, Brolo AG (2011) A review on the fabrication of substrates for surface enhanced Raman spectroscopy and their applications in analytical chemistry. Anal Chim Acta 693:7-25. https://doi.org/10.1016/j.aca.2011.03.002

17. Cardinal MF, Vander Ende E, Hackler RA, et al (2017) Expanding applications of SERS through versatile nanomaterials engineering. Chem Soc Rev 46:3886-3903. https://doi.org/10.1039/c7cs00207f

18. Lafuente M, Berenschot EJW, Tiggelaar RM, et al (2018) 3D fractals as SERS active platforms: Preparation and evaluation for gas phase detection of G-nerve agents. Micromachines 9:. https://doi.org/10.3390/mi9020060

19. Berenschot EJW, Jansen H V., Tas NR (2013) Fabrication of 3D fractal structures using nanoscale anisotropic etching of single crystalline silicon. $\mathrm{J}$ Micromechanics Microengineering 23:. https://doi.org/10.1088/0960-1317/23/5/055024

20. Le Ru EC, Blackie E, Meyer M, Etchegoint PG (2007) Surface enhanced raman scattering enhancement factors: A comprehensive study. J Phys Chem C 111:1379413803. https://doi.org/10.1021/jp0687908

21. Rodrigo SG (2012) Optical Properties of Nanostructured Metallic Systems. Springer Science \& Business Media.

22. Rodrigo SG, García-Vidal FJ, Martín-Moreno L (2008) Influence of material properties on extraordinary optical transmission through hole arrays. Phys Rev B - Condens Matter Mater Phys 77:1-8. https://doi.org/10.1103/PhysRevB.77.075401

23. Fathi F, Lagugné-Labarthet F, Pedersen DB, Kraatz HB (2012) Studies of the interaction of two organophosphonates with nanostructured silver surfaces. Analyst 137:4448-4453. https://doi.org/10.1039/c2an35641d

24. El Alami A, Lagarde F, Tamer U, et al (2016) Enhanced Raman spectroscopy coupled to chemometrics for identification and quantification of acetylcholinesterase inhibitors. Vib Spectrosc 87:27-33. https://doi.org/10.1016/j.vibspec.2016.09.005

25. Ma B, Li P, Yang L, Liu J (2015) Based on time and spatial-resolved SERS mapping strategies for detection of pesticides. Talanta 141:1-7. https://doi.org/10.1016/j.talanta.2015.03.053

26. Li P, Dong R, Wu Y, et al (2014) Polystyrene/Ag nanoparticles as dynamic surfaceenhanced Raman spectroscopy substrates for sensitive detection of organophosphorus pesticides. Talanta 127:269-275. https://doi.org/10.1016/j.talanta.2014.03.075 
27. Wang B, Zhang L, Zhou X (2014) Synthesis of silver nanocubes as a SERS substrate for the determination of pesticide paraoxon and thiram. Spectrochim Acta - Part A Mol Biomol Spectrosc 121:63-69. https://doi.org/10.1016/j.saa.2013.10.013

28. Jiang Y, Wang J, Malfatti L, et al (2018) Highly durable graphene-mediated surface enhanced Raman scattering (G-SERS) nanocomposites for molecular detection. Appl Surf Sci 450:451-460. https://doi.org/10.1016/j.apsusc.2018.04.218

29. Scalora M, Vincenti MA, de Ceglia D, et al (2012) Raman scattering near metal nanostructures. J Opt Soc Am B 29:2035. https://doi.org/10.1364/josab.29.002035

30. Moreno E, Garcia-Vidal F, Rodrigo SG, Martin-Moreno L (2009) Fundamentals of channel and wedge plasmon polaritons. In: Plasmonics: nanoguides and circuits. Pan Stanford Publishing, Singapore, pp 253-272

31. Yaakobi K, Liebes-Peer Y, Kushmaro A, Rapaport H (2013) Designed amphiphilic $\beta$ sheet peptides as templates for paraoxon adsorption and detection. Langmuir 29:68406848. https://doi.org/10.1021/la401280e 


\title{
Attomolar SERS detection of organophosphorous pesticides using silver mirror like micro-pyramids as active substrate
}

\author{
Marta Lafuente ${ }^{1,2}$, Erwin J. W. Berenschot ${ }^{3}$, Roald M. Tiggelaar ${ }^{4}$, Sergio G. Rodrigo ${ }^{2,5}$,
} Reyes Mallada $^{1,2}$, Niels R. Tas ${ }^{3}$, María P. Pina ${ }^{1,2}$

${ }^{1}$ Instituto de Nanociencia de Aragón (INA), Departamento de Ingeniería Química y Tecnología del Medio Ambiente. Universidad de Zaragoza, 50018, Zaragoza, Spain.

${ }^{2}$ Instituto de Ciencia de los Materiales de Aragón (ICMA), CSIC-Universidad de Zaragoza, 50009, Zaragoza, Spain.

${ }^{3}$ Mesoscale Chemical Systems, MESA+ Institute for Nanotechnology, University of Twente, P.O. Box 217, 7500 AE Enschede, The Netherlands.

${ }^{4}$ MESA+ NanoLab cleanroom, MESA+ Institute for Nanotechnology, University of Twente, P.O. Box 217, 7500 AE Enschede, The Netherlands

${ }^{5}$ Centro Universitario de la Defensa, Carretera Huesca s/n, 50090 Zaragoza, Spain.

María P. Pina, mapina@unizar.es, +34 976751155. 


\section{S1. Calculation of Analytical Enhancement Factor (AEF)}

The AEF provides quantitative information of the signal enhancement that we could expect from a specific SERS substrate with respect to a reference Raman experiment. The 4nitrobenzenethiol (4-NBT) was selected as probe molecule, due to the well-known affinity of thiol group towards $\mathrm{Ag}$ surface. monitoring its $\mathrm{NO}_{2}$ symmetric stretching vibrational mode displayed at $1338 \mathrm{~cm}^{-1}$. AEF was calculated using Equation $\mathbf{S 1}$ as follows:

$$
A E F=\frac{\frac{I_{S E R S}}{C_{S E R S}}}{\frac{I_{\text {Raman }}}{C_{\text {Raman }}}}
$$

where $\mathrm{C}_{\text {Raman }}$ and $\mathrm{C}_{\text {SERS }}$ are the 4-NBT concentration in the Raman measurements and SERS conditions, respectively. The normal Raman 4-NBT spectrum was measured focusing the laser beam inside the stock solution $9 \times 10^{-3} \mathrm{M}$ of $4-\mathrm{NBT}$. Thus, $\mathrm{I}_{\text {Raman }}$ corresponds to the intensity of the 4-NBT molecules measured in liquid phase. For SERS measurements, the substrates were incubated in $1 \times 10^{-6} \mathrm{M}$ of 4-NBT, prepared from the mother solution, in vertical position for 1 hour; then, they were rinsed in ethanol and air-dried. Thus, ISERS is the intensity of the 4-NBT molecules adsorbed in the SERS substrate. For this work, the SERS spectra of the substrates were measured in five different random areas (10×10 excitations points/area) and the intensity of the peak at $1338 \mathrm{~cm}^{-1}$ was averaged.

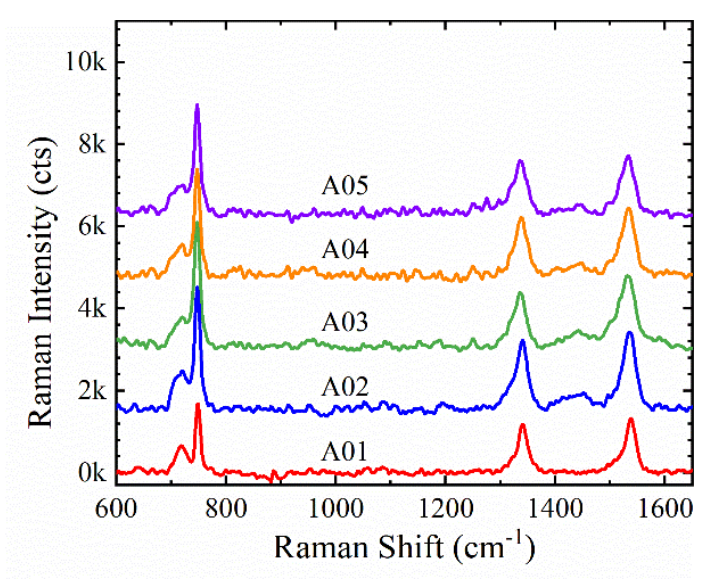

Figure S1. Average SERS spectra of 4-NBT $10^{-6} \mathrm{M}$ obtained in five different areas randomly selected. 
S2. SEM and TEM images of continuous Ag films on 3D G0 and G1 type substrates

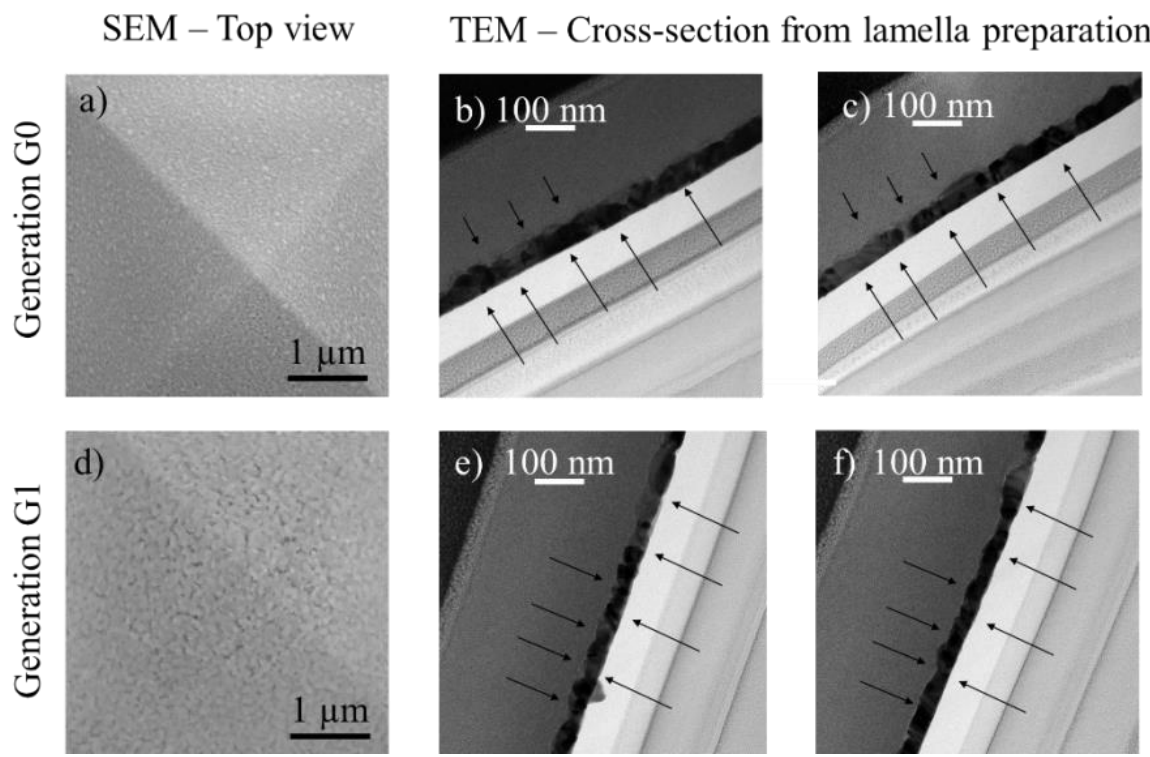

Figure S2. (a) SEM and (b-c) TEM images of G0 type substrates. (d) SEM and (e-f) TEM images of G1 type substrates.

\section{TEM picture of lamella extraction-fabrication:}

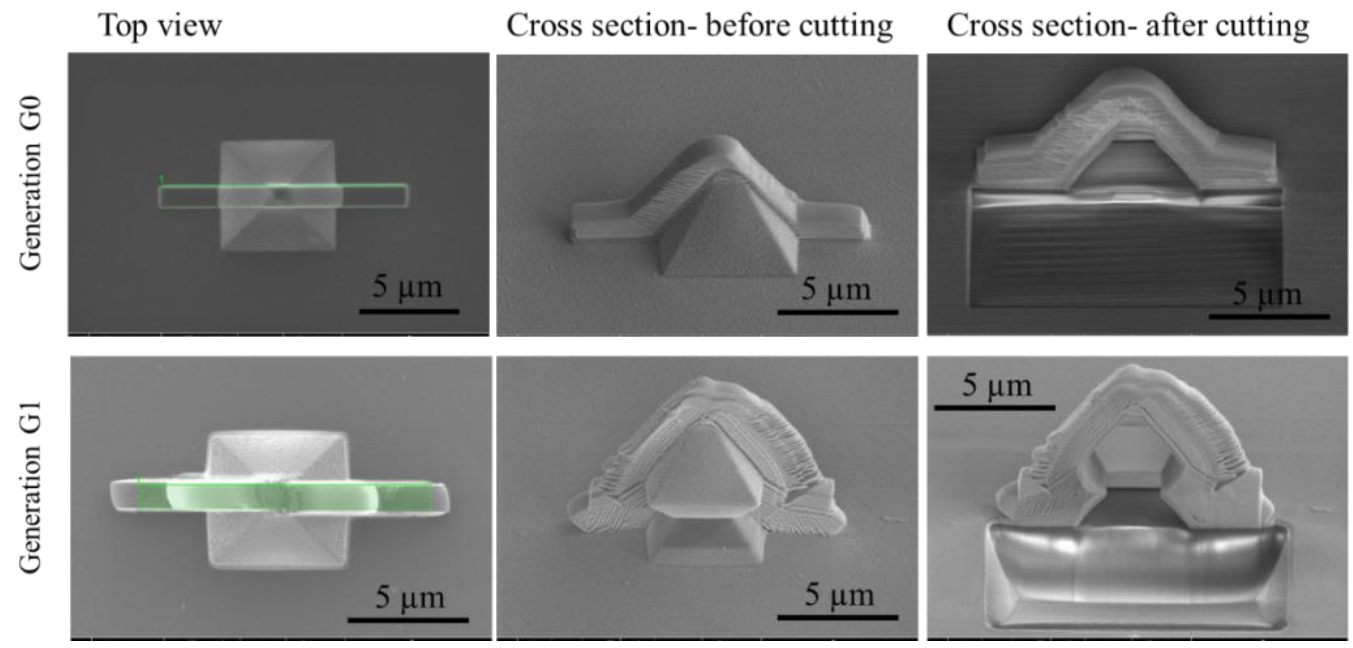




\section{S3. Paraoxon Molecules in the tested solutions and scattered by the laser beam.}

Calculations for $10^{-18} \mathrm{M}$ probe solution

$$
\begin{aligned}
C\left(\frac{\mathrm{mol}}{L}\right) \cdot \text { Volume }_{\text {incubation solution }}(L) \cdot \text { Avogadros's number }\left(\frac{\text { molecules }}{\mathrm{mol}}\right) \\
\quad=1 \times 10^{-18}\left(\frac{\mathrm{mol}}{\mathrm{L}}\right) \cdot 0.025 \mathrm{~L} \cdot 6.023 \times 10^{23}\left(\frac{\text { molecules }}{\mathrm{mol}}\right) \\
=\mathbf{1 5 0 5 7} \text { molecules }
\end{aligned}
$$

Distribution of pesticides molecules on silver-mirror like 3D G1 type substrate.

Total area of the SERS substrate $\rightarrow 0.2 \mathrm{~cm}^{2}$

2D-Mapping (area) $\rightarrow 200 \mu \mathrm{m} \times 150 \mu \mathrm{m}=3 \times 10^{4} \mu \mathrm{m}^{2}$

Assuming an homogeneous distribution of Paraoxon molecules over the substrate

$$
\begin{gathered}
\qquad \text { o areas }=\frac{\text { Area substrate }}{\text { area mapping }}=\frac{0.2 \times 10^{8} \mu \mathrm{m}^{2}}{3 \times 10^{4} \mu \mathrm{m}^{2}}=666 \text { areas } \\
\frac{\text { Total molecules in the incubation solution }}{\text { number of areas in the substrate }}=\frac{15057 \text { molecules }}{666 \text { areas }}=23 \frac{\text { molecules }}{\text { area }}
\end{gathered}
$$

Estimation of Surface Coverage Degree by Paraoxon molecules

2D-Mapping Area $\rightarrow 23$ molecules and 77 micropyramids

Cross-section of paraoxon-methyl molecule $=0.2 \mathrm{~nm}^{2}$

Assuming an hexagonal lattice for the ordered pesticide molecules arrangement on the Ag surface $\rightarrow 0.65 \mathrm{~nm}^{2}$ per molecule

Assuming that the hot spots are located on the pyramidal edges where edge plasmon polaritons are excited $\rightarrow 0.08 \mu \mathrm{m}^{2}$ of hot spots per pyramid $\rightarrow 6.16 \mu \mathrm{m}^{2}$ of hot spots per mapping area

$$
\begin{gathered}
\frac{\text { Total molecules located in the vecinity of hot spots }}{\text { scanned area }}=\frac{6.16 \times 10^{6} \mathrm{~nm}^{2}}{0.65 \mathrm{~nm}^{2}} \\
=9.47 \times 10^{6} \frac{\text { molecules }}{\text { EPPs area }}
\end{gathered}
$$

The lateral area of the pyramids where the breathing modes are excited $\rightarrow 67.6 \mu \mathrm{m}^{2}$ per pyramid $\rightarrow 5.20 \times 10^{3} \mu \mathrm{m}^{2}$ of pyramidal faces per mapping area 


$$
\begin{aligned}
& \frac{\text { Total molecules located in the pyramidal faces }}{\text { scanned area }}=\frac{5.20 \times 10^{9} \mathrm{~nm}^{2}}{0.65 \mathrm{~nm}^{2}} \\
& =8.01 \times 10^{9} \frac{\text { molecules }}{\text { breathing modes area }}
\end{aligned}
$$

Accordingly, the edges and pyramidal faces are fully coveraged by a monolayer of pesticides molecules, assuming an ideal hexagonal compact arrangement, for $3.5 \times 10-{ }^{10} \mathrm{M}$ paraoxon concentration. For higher paraoxon concentration values, we hypothesize that the increased number of molecules in solution becomes preferentially adsorbed on the planar 2D surface. This behaviour would be supporting the existence of the two observed distinctive regions when analysing the SERS intensity as a function of pesticide concentration. The transition between region I and region II takes place at concentrations higher than $10^{-12} \mathrm{M}$, in agreement with the complete formation of a monolayer on the lateral surface area of the micropyramids.

\begin{tabular}{c|ccc}
$\begin{array}{c}\text { Concentration of the } \\
\text { Incubation solution (M) }\end{array}$ & $\begin{array}{c}\text { Paraoxon } \\
\text { molecules per } \\
\text { scanned area }\end{array}$ & $\begin{array}{c}\text { Surface coverage of } \\
\text { pyramidal edges (\%) }\end{array}$ & $\begin{array}{c}\text { Surface coverage of } \\
\text { pyramidal faces (\%) }\end{array}$ \\
\hline $10^{-6} \mathrm{M}$ & $23 \times 10^{12}$ & 100 & 100 \\
$10^{-9} \mathrm{M}$ & $23 \times 10^{9}$ & 100 & 100 \\
$10^{-12} \mathrm{M}$ & $23 \times 10^{6}$ & 100 & 0.17 \\
$10^{-15} \mathrm{M}$ & $23 \times 10^{3}$ & $2.42 \times 10^{-1}$ & 0 \\
$10^{-18} \mathrm{M}$ & 23 & $2.42 \times 10^{-4}$ & 0
\end{tabular}




\section{S4. SERS measurements on G1 type substrates incubated with Paraoxon}

RSD values for SERS measurements as a function of Paraoxon concentration

\begin{tabular}{c|cc|cc|}
\multirow{2}{*}{$\begin{array}{c}\text { Concentration } \\
(\mathrm{mol} / \mathrm{L})\end{array}$} & \multicolumn{2}{|c|}{ Peak at $746 \mathrm{~cm}^{-1}$} & \multicolumn{2}{c|}{ Peak at $1338 \mathrm{~cm}^{-1}$} \\
\cline { 2 - 5 } & $\begin{array}{c}\text { AVG } \pm \text { Stand. Dev } \\
\text { (cts) }\end{array}$ & RSD (\%) & $\begin{array}{c}\text { AVG } \pm \text { Stand. Dev } \\
\text { (cts) }\end{array}$ & RSD (\%) \\
\hline $1 \times 10^{-6}$ & $1644 \pm 180$ & 11 & $925 \pm 104$ & 11 \\
$1 \times 10^{-9}$ & $1586 \pm 181$ & 11 & $892 \pm 52$ & 6 \\
$1 \times 10^{-12}$ & $1408 \pm 288$ & 20 & $767 \pm 93$ & 12 \\
$1 \times 10^{-15}$ & $1080 \pm 201$ & 19 & $568 \pm 69$ & 12 \\
$1 \times 10^{-18}$ & $964 \pm 247$ & 26 & $485 \pm 115$ & 24
\end{tabular}

The differences found in RSD is associated with the existence of two different regions as explained in previous section S3. Thus, the adsorption of the molecules occurs in different areas. In region I, i.e. at concentrations below $10^{-12} \mathrm{M}$, paraoxon molecules are mainly allocated on the edges and pyramidal faces. Within this region, the registered RSD values are higher because slight variations on the molecules arrangement on the metal surface provoke a remarkable effect on the SERS intensity due to they are being preferentially adsorbed on the hot spots. On the contrary, in region II, at concentrations clearly above $10^{-12} \mathrm{M}$, the registered RSD values are lower because the paraoxon molecules are mainly allocated on the colder planar 2D surface which are contributing in much less extent to the total registered SERS signal. 